\title{
Quantitative evaluation of grain boundary activity in multicrystalline semiconductors by light beam induced current: An advanced model
}

\author{
G. Micard, ${ }^{\text {a) }}$ G. Hahn, A. Zuschlag, S. Seren, and B. Terheiden \\ Department of Physics, University of Konstanz, P.O. Box X916, 78457 Konstanz, Germany
}

(Received 23 February 2010; accepted 11 June 2010; published online 11 August 2010)

\begin{abstract}
We present an advanced analytical model which applies to light beam induced current contrast profiles to determine reliably the effective surface recombination velocities $\left(S_{\text {eff }}\right)$ of grain boundaries $(\mathrm{GBs})$ and diffusion lengths $\left(\mathrm{L}_{\text {diff }}\right)$ in the grains, in cases where a GB is close to the studied one or when $\mathrm{L}_{\text {diff }}$ of the neighboring grain differs. We introduce additionally a new method for a very accurate determination of the plateau value of the investigated linescan and make use of simultaneously fitting GB profiles measured at various laser wavelengths both aiming at increasing the accuracy of the $\mathrm{L}_{\text {diff }}$ determination. Through several special case investigations, the various applications and limitations of the model are demonstrated. We discuss the influence of the electrical parameters of the semiconductor on the various zones of the profile as well as the influence of measurement technique parameters on the experimental profile and point out the need of an accurately determined small laser beam radius to ensure a reliable extraction of $S_{\text {eff }}$. We discuss the occurring discrepancy between fit and measured data and show that it gives hints about particular material features and the reliability of the extracted parameters. We finally point out the possibility of determining $\mathrm{L}_{\text {diff }}$ in small grains. This model thus allows more realistic GB situations to be investigated. (C) 2010 American Institute of Physics. [doi:10.1063/1.3462447]
\end{abstract}

\section{INTRODUCTION}

Multicrystalline semiconductors are of major interest for low cost and large size electronic applications. The main reason for the lower performance of multicrystalline semiconductor devices in comparison to monocrystalline ones is the detrimental influence of the grain boundaries (GBs). The GBs affect the performance directly by a current and voltage drop due to recombination or indirectly by a voltage drop induced by local fixed charges. Therefore, semiconductor GBs have been extensively characterized ${ }^{1-3}$ and the transport properties have shown to involve complex phenomena (e.g., an injection dependant potential barrier induced by charged defects at the GB influencing its recombination strength ${ }^{4}$ ). A complete study therefore involves several characterization techniques on one isolated GB (e.g., capacitance voltage measurements on a silicon bicrystal ${ }^{1}$ ).

In general, a GB can hardly be considered isolated in real multicrystalline materials and therefore spatially resolved measurement techniques like light beam induced current (LBIC) or electron beam induced current (EBIC) are often used for a quantitative analysis. As only the overall result of all the different phenomena occurring at the GB can be straightforwardly investigated using LBIC or EBIC measurements, the effective surface recombination velocity $\left(\mathrm{S}_{\text {eff }}\right)$ is the only GB parameter that can be extracted exploiting these measurements. By reducing the GB to a discrete surface affected by $S_{\text {eff }}$, the advanced model described in this contribution allows its reliable extraction for various GB arrangements encountered in real multicrystalline materials.

In the case of, e.g., multicrystalline silicon based solar

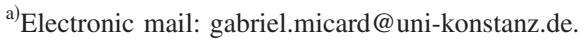

cells, relevant models for LBIC or EBIC investigations are based on particular solutions of the steady state minority carrier diffusion equation only. These models allow determining $S_{\text {eff }}$ of the GB and the minority carrier diffusion length $\left(\mathrm{L}_{\text {diff }}\right)$ of the neighboring grains by fitting the GB contrast profile. The GB contrast profile is a linescan perpendicular to the GB extracted from LBIC or EBIC maps normalized to the level obtained infinitively far from the GB (also called plateau level). In this framework, Donolato ${ }^{5}$ developed a model for the simple case when the studied GB can be considered to be isolated.

It has, however, been remarked by von Roos and Luke ${ }^{6,7}$ that different $L_{\text {diff }}$ on either side of the GB as well as the presence of another GB located at less than $4 \mathrm{~L}_{\text {diff }}$ from the studied GB can induce strong asymmetry in the profile leading to wrong estimations of $\mathrm{L}_{\text {diff }}$ and $\mathrm{S}_{\text {eff. }}$. It has been remarked by Corkish et al. ${ }^{8}$ that the method of Donolato, ${ }^{5}$ that estimates $L_{\text {diff }}$ and $S_{\text {eff }}$ through the variance and the area of the dip in the contrast profile, leads to errors in the deduced parameters because local inhomogeneities in the grains are not properly considered. Additionally, it has been demonstrated that estimating the variance accurately requires to extract a GB profile spreading over a distance of several $L_{\text {diff }}$ from the GB. ${ }^{8}$ In order to neglect the influence of a second $\mathrm{GB}$, the grain size should be approximately twice this aforementioned distance. For this reason, only grains that are very large compared to $\mathrm{L}_{\text {diff }}$ can be investigated and thus only few GB situations can be investigated reliably with this method. Thus, Corkish et $_{\mathrm{al} .}{ }^{8}$ proposed a direct fitting procedure as a more reliable method.

These mostly theoretical studies were dedicated mainly to the evaluation of EBIC contrast profiles. While EBIC presents the advantage of a very localized spatial distribution of 


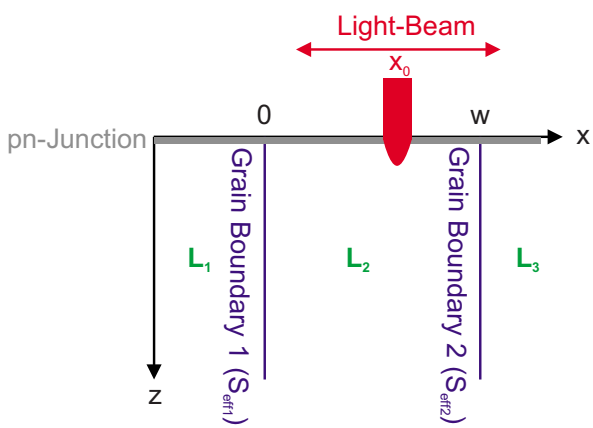

FIG. 1. (Color online) Schematic of the diffusion problem.

the generated electron hole pairs [with a maximum located close to but significantly below the pn-junction using a beam energy of $30 \mathrm{keV}$ (Ref. 5)], LBIC is a much more widespread technique in solar cell laboratories than EBIC. We, therefore, present here an advanced model adapted for LBIC contrast profiles that includes two GBs arbitrarily spaced and with arbitrary diffusion length in the three regions in which the semiconductor is divided by the two GBs.

We chose to develop an analytical solution because it allows the visualization of the different influences of each element (GBs, $\mathrm{L}_{\text {diff }}$ in the grains) in the profile equation for selected special cases. In addition, we like to point out that the solution should fulfill strong requirements in terms of speed (trial and error methods as well as optimizations algorithms require several evaluations of the solution) and accuracy (less than $0.1 \%$ error is required). These two requirements are far better met by an optimized algorithm computing an analytical solution than by a complete numerical simulation even very well optimized.

First the model and its assumptions are given. Then, we describe important implementation issues regarding numerical computation convergence as well as accurate determination of the plateau level. We explain the applicability limits of the model by demonstrating the evaluation procedure with some examples. In the end, we will present some further extensions regarding improving accuracy, robustness and applicability of the method.

\section{MODEL DESCRIPTION}

\section{A. Assumptions}

The presented model is a two-dimensional reduction in the $(\mathrm{x}, \mathrm{z})$ plane of the three-dimensional problem. It is therefore assumed that the semiconductor is invariant along the y-axis.

The configuration on which the model is based is shown in Fig. 1. Two GBs, named GB1 and GB2, with respective effective surface recombination velocity $\mathrm{S}_{\text {eff1 }}$ and $\mathrm{S}_{\text {eff2 }}$ separated by a distance $\mathrm{w}$, divide the semiconductor in three regions with minority carrier diffusion length $\mathrm{L}_{1}, \mathrm{~L}_{2}$, and $\mathrm{L}_{3}$.

The current collection is performed by the pn-junction with the boundary condition of zero excess carrier density. Within this approximation, the carriers generated in its space charge region (SCR) are assumed to be negligible in comparison to the ones generated in the bulk. This assumption requires choosing the laser wavelength so that the light penetration depth in silicon is much larger than the SCR width.
It is further assumed that the diffusion length is homogeneous in each region and the GBs are oriented perpendicular to the wafer surface. GBs are described as discrete surfaces affected by an effective surface recombination velocity.

It is assumed also that the laser beam has a Gaussian power density distribution and its reflection at the surface is constant over the whole linescan. In addition, the incident beam is normal to the semiconductor surface and does not undergo multiple surface reflections.

It is further assumed that the finite thickness and the back surface recombination have only a small impact on the fitted profile shape. Therefore this theory is established considering an infinite thickness of the multicrystalline material. This approximation requires that the light penetration depth in silicon and $\mathrm{L}_{\text {diff }}$ are smaller than the wafer thickness.

\section{B. Model}

For an n-doped wafer based solar cell in low injection condition, the minority excess carrier density $\mathrm{p}(\mathrm{r})$ diffusion equation in steady state is expressed in Eq. (1) using Donolato's formalism: ${ }^{5}$

$$
\begin{aligned}
\mathrm{D}_{\mathrm{p}} \Delta \mathrm{p}(\mathrm{r})-\frac{1}{\tau_{\mathrm{p}}} \mathrm{p}(\mathrm{r})= & -\mathrm{g}(\mathrm{r}) \quad \text { or } \quad \Delta \mathrm{p}(\mathrm{r})-\Lambda^{2} \mathrm{p}(\mathrm{r})= \\
& -\frac{\mathrm{g}(\mathrm{r})}{\mathrm{D}_{\mathrm{p}}},
\end{aligned}
$$

where $\mathrm{D}_{\mathrm{p}}$ denotes the minority carrier diffusion constant, $\tau_{\mathrm{p}}$ their lifetime, $\Lambda=1 / \mathrm{L}_{\text {diff }}=1 /\left(\mathrm{D}_{\mathrm{p}} \times \tau_{\mathrm{p}}\right)^{1 / 2}$ is the inverse of the diffusion length $\mathrm{L}_{\text {diff, }} \mathrm{g}(\mathrm{r})$ the spatial distribution of the generated electron hole pairs induced by a source (electron or light beam) also called the volume generation function and $\mathrm{r}$ the position that could be expressed in three-dimensional Cartesian coordinates by $\mathrm{x}, \mathrm{y}$, and $\mathrm{z}$. Therefore, the problem can be schematically represented according to Fig. 1 in which the junction is represented as an infinitely recombinative surface with

$$
\left.\mathrm{p}(\mathrm{r})\right|_{\mathrm{z}=0}=0 .
$$

At the GBs, a first condition expressed by Eqs. (3a) and (3b) imposes the continuity of the carrier concentration.

$$
\left.\mathrm{p}(\mathrm{r})\right|_{\mathrm{x}=0^{+}}=\left.\mathrm{p}(\mathrm{r})\right|_{\mathrm{x}=0^{-}}
$$

and

$$
\left.\mathrm{p}(\mathrm{r})\right|_{\mathrm{x}=\mathrm{w}^{+}}=\left.\mathrm{p}(\mathrm{r})\right|_{\mathrm{x}=\mathrm{w}^{-}}
$$

The second condition [Eqs. (3c) and (3d)] imposes that the total minority carrier flux (total recombination current) at the GBs is proportional to the local carrier density

$$
\left.\frac{\partial \mathrm{p}}{\partial \mathrm{x}}\right|_{\mathrm{x}=0^{+}}-\left.\frac{\partial \mathrm{p}}{\partial \mathrm{x}}\right|_{\mathrm{x}=0^{-}}=\left.\mathrm{s}_{1} \mathrm{p}(\mathrm{r})\right|_{\mathrm{x}=0}
$$

and

$$
\left.\frac{\partial \mathrm{p}}{\partial \mathrm{x}}\right|_{\mathrm{x}=\mathrm{w}^{+}}-\left.\frac{\partial \mathrm{p}}{\partial \mathrm{x}}\right|_{\mathrm{x}=\mathrm{w}^{-}}=\left.\mathrm{s}_{2} \mathrm{p}(\mathrm{r})\right|_{\mathrm{x}=\mathrm{w}}
$$

introducing $\mathrm{s}_{1,2}$ the reduced surface recombination velocity at the GBs corresponding to 


$$
\mathrm{s}_{1,2}=\frac{\mathrm{S}_{\mathrm{eff} 1,2}}{\mathrm{D}_{\mathrm{p}}}
$$

with $\mathrm{S}_{\text {eff } 1,2}$ the effective surface recombination velocity at GB1 and GB2.

Considering that an LBIC measurement uses a Gaussian laser beam, the volume generation function $\mathrm{g}(\mathrm{r})$ in Eq. (1) can be expressed as

$$
\mathrm{g}(\mathrm{r})=\mathrm{g}_{0} \times \exp \left(-\frac{\mathrm{x}^{2}+\mathrm{y}^{2}}{\sigma^{2}}\right) \times \exp (-\alpha \mathrm{z}) \text {. }
$$

In which the prefactor $\mathrm{g}_{0}$ is expressed as

$$
\mathrm{g}_{0}=\Phi \alpha \eta(1-\mathrm{R}) \text {. }
$$

With $\Phi$ the maximum photon flux density of the laser beam (photons $\mathrm{cm}^{-2} \mathrm{~s}^{-1}$ ), $\alpha$ the absorption coefficient for the laser wavelength, $\eta$ the quantum efficiency, $\mathrm{R}$ the reflection coefficient, and $\sigma$ the beam radius defined as the radius where the amplitude has dropped by a factor $1 / \mathrm{e}$.

Then the measured current collected at the junction is found by integrating the normal gradient of $\mathrm{p}(\mathrm{r})$ [solution of Eq. (1) with boundary conditions of Eqs. (2) and (3a)-(3d)] at the surface plane $\mathrm{z}=0$ times the elementary charge $q$

$$
I=\left.q D_{p} \int_{-\infty}^{\infty} \int_{-\infty}^{\infty} \frac{\partial p}{\partial z}\right|_{z=0} d x d y .
$$

Using the Green's function method, Donolato shows that the collected current can be obtained by the solution of a twodimensional partial differential equation (PDE) for the twodimensional Green's function $G$ instead of the threedimensional PDE of the minority carrier diffusion given by Eq. (1), providing an invariance of the problem along the y-axis. ${ }^{5}$ As the derivation of the formula finally used for fitting the profiles is quite lengthy, we will present only the end result here, while the most important steps leading to the final formula presented in Eq. (8) are presented in Appendix A.

Considering only one diffusion length for the whole problem, Donolato shows that his GB linescan model equation is the sum of a negative term corresponding to the GB recombination current and of a constant background current term corresponding to the current obtained very far from or without a GB, the so called plateau level. This additive constant depends on the reflectance of the multicrystalline wafer surface at the laser wavelength $(R)$ and on the maximum photon flux density of the laser beam $(\Phi)$ through the factor $\mathrm{g}_{0}$ [see Eq. (6)] as well as of the laser beam radius $(\sigma)$ and of the diffusion length $\left(\mathrm{L}_{\text {diff }}\right)$, which is constant in Donolato's case, and therefore its accuracy is limited by the uncertainty associated with the determination of these parameters. He then decided to remove this constant by subtracting it and to normalize the remaining expression by this term. This is the so called contrast profile which is independent of this value. However, in our model the background current term [see Eq. (A22)] depends on the laser beam position $\left(\mathrm{x}_{0}\right)$ because of the different diffusion lengths of each region that influence its value. Therefore, this term cannot be removed straightforwardly from the equation. One can nevertheless remove the constant $\mathrm{g}_{0}$ by normalizing the expression of the collected current to the plateau level obtained on the left side of the profile $\left(\mathrm{I}_{01}\right)$. Doing so, we remove the dependence in $\mathrm{A}$ and $\mathrm{R}$ but, however, we keep the dependence in $\sigma$ and $\mathrm{L}_{1,2,3}$. We have to point out that the choice of the left side is arbitrary, and the right side could have been taken instead.

The contrast profile normalized to the left plateau $\mathrm{I}_{01}$ is defined by Eq. (8)

$$
\begin{aligned}
& \frac{I\left(x_{0}\right)}{I_{01}}=\frac{1}{2}\left[2-\left(\frac{\Lambda_{2}-\Lambda_{1}}{\alpha+\Lambda_{2}}\right) \operatorname{erfc}\left(-\frac{x_{0}}{\sigma}\right)-\left(\frac{\alpha+\Lambda_{1}}{\alpha+\Lambda_{2}}\right)\left(\frac{\Lambda_{3}-\Lambda_{2}}{\alpha+\Lambda_{3}}\right) \operatorname{erfc}\left(\frac{w-x_{0}}{\sigma}\right)\right] \\
& -\frac{1}{\pi}\left(\alpha+\Lambda_{1}\right) \times \int_{0}^{\infty} \frac{k^{2}}{\mu_{1} \mu_{2} \mu_{3}\left[\left(s_{1}+\mu_{1}+\mu_{2}\right)\left(s_{2}+\mu_{2}+\mu_{3}\right)-e^{-2 \mu_{2} w}\left(s_{1}+\mu_{1}-\mu_{2}\right)\left(s_{2}+\mu_{3}-\mu_{2}\right)\right] \times\left(k^{2}+\alpha^{2}\right)} \\
& {\left[\frac{e^{\left(\frac{\mu_{1} \sigma}{2}\right)^{2}}}{\mu_{1}}\left\{\begin{array}{c}
\mu_{3}\left(s_{2}+\mu_{3}+\mu_{2}\right)\left[\mu_{2}\left(\mu_{2}+s_{1}\right)-\mu_{1}^{2}\right] \\
+2 e^{-\mu_{2} w} \mu_{1}^{2}\left[\mu_{3}\left(\mu_{3}+s_{2}\right)-\mu_{2}^{2}\right] \\
+e^{-2 \mu_{2} w} \mu_{3}\left(s_{2}+\mu_{3}-\mu_{2}\right)\left[\mu_{2}\left(\mu_{2}-s_{1}\right)-\mu_{1}^{2}\right]
\end{array}\right\} \times e^{\mu_{1} x_{0}} \operatorname{erfc}\left(\frac{\mu_{1} \sigma}{2}+\frac{x_{0}}{\sigma}\right)\right.}
\end{aligned}
$$

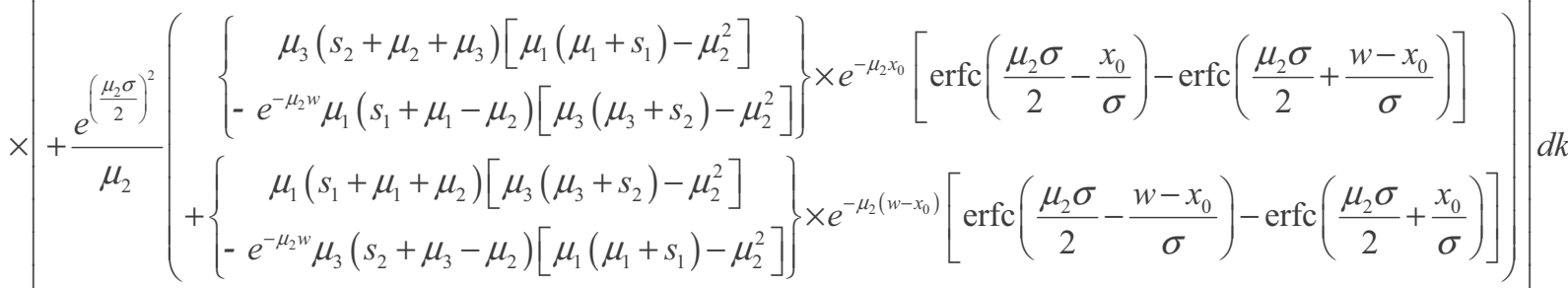

$$
\begin{aligned}
& +\frac{e^{\left(\frac{\mu_{3} \sigma}{2}\right)^{2}}}{\mu_{3}}\left\{\begin{array}{c}
\mu_{1}\left(s_{1}+\mu_{1}+\mu_{2}\right)\left[\mu_{2}\left(\mu_{2}+s_{2}\right)-\mu_{3}^{2}\right] \\
+2 e^{-\mu_{2} w} \mu_{3}^{2}\left[\mu_{1}\left(\mu_{1}+s_{1}\right)-\mu_{2}^{2}\right] \\
+e^{-2 \mu_{2} w} \mu_{1}\left(s_{1}+\mu_{1}-\mu_{2}\right)\left[\mu_{2}\left(\mu_{2}-s_{2}\right)-\mu_{3}^{2}\right]
\end{array}\right\} \times e^{\mu_{3}\left(w-x_{0}\right)} \operatorname{erfc}\left(\frac{\mu_{3} \sigma}{2}+\frac{w-x_{0}}{\sigma}\right)
\end{aligned}
$$


With $\mu_{1,2,3}=\left(\mathrm{L}_{1,2,3}^{-2}+\mathrm{k}^{2}\right)^{1 / 2}=\left(\Lambda_{1,2,3}^{2}+\mathrm{k}^{2}\right)^{1 / 2}, \mathrm{x}_{0}$, the position of the center of the laser referring to the GB1 position and $\mathrm{k}$ an integration constant.

In order to keep the equations simple, we avoid conversion factors where possible. Therefore, Eq. (8) is expressed as a function of $\sigma$ and not the full width half maximum (FWHM) of the laser beam. The conversion is, however, straightforward

$$
\mathrm{FWHM}=2 \sqrt{\ln (2)} \times \sigma \approx 1.665 \times \sigma .
$$

\section{Implementation}

We created a code in MATLAB to use Eq. (8) as a fitting expression on a measured LBIC profile linescan over a GB and normalized to the left side plateau level in which $\mathrm{L}_{1}, \mathrm{~L}_{2}, \mathrm{~L}_{3}$ and $\mathrm{s}_{1}, \mathrm{~s}_{2}$ are fitting parameters. Due to some critical aspects mostly related to plateau level evaluations as detailed in Sec. IV B we observed that standard optimization methods, like e.g., the Nelder and Mead simplex or the Levenberg-Marquardt algorithm, lead to unrealistic values of the diffusion length for high diffusion lengths. We therefore present a trial and error method in this work.

The evaluation of the " $\exp \left(\mathrm{y}^{2}\right) \times \operatorname{erfc}(\mathrm{y})$ " terms in Eq. (8) leads to very strong divergences if inadequately handled when y tends to infinity (our case). Indeed, mathematically speaking $\exp \left(\mathrm{y}^{2}\right)$ will tend very quickly to infinity while erfc(y) will tend to 0 slightly more quickly making the global result tending slowly to 0 . However, computationally speaking, this term leads to a "not a number" resulting from the operation " $\infty \times 0$ " even when y is not very large because of the very strong variation in both functions. An adequate way to treat these terms computationally is described in Appendix B.

The numerical evaluation of the improper integral in Eq. (8) was performed most efficiently using the Gauss-Kronrod quadrature. $^{9}$

As we normalize the first plateau (left side) to 1 by convention, this operation implies that the plateau level of regions 2 and 3 are expressed according to Eq. (10).

$$
\mathrm{P}_{2,3}=\frac{\alpha+\Lambda_{1}}{\alpha+\Lambda_{2,3}} .
$$

As $\mathrm{P}_{2,3}$ depends on $\mathrm{L}_{1}$ and $\mathrm{L}_{2,3}$, a variation in one or the other diffusion length influences it. However, $\mathrm{P}_{2}$ and $\mathrm{P}_{3}$ are straightforwardly extractable quantities corresponding to slight overestimations of the maximum value observed in the respective region on the normalized profile. Using Eq. (10), we can express $L_{2,3}$ as a function of $L_{1}$ and $P_{2,3}$

$$
\mathrm{L}_{2,3}=\frac{\mathrm{L}_{1} \mathrm{P}_{2,3}}{1+\alpha \mathrm{L}_{1}\left(1-\mathrm{P}_{2,3}\right)}
$$

Therefore, the determination of $\mathrm{L}_{1,2,3}$ is equivalent to the determination of $\mathrm{L}_{1}$ and $\mathrm{P}_{2,3}$ making use of Eq. (11). However, as $\mathrm{P}_{2,3}$ are straightforwardly extractable for the reason mentioned above, only $\mathrm{L}_{1}$ remains as nonstraightforwardly extractible parameter instead of $\mathrm{L}_{1,2,3}$ in the direct approach.
This procedure therefore simplifies the determination of the diffusion lengths when they differ.

It is worth mentioning that $\mathrm{P}_{2}$ and $\mathrm{P}_{3}$ have a maximum value of $\mathrm{P}_{\max }=1+1 /\left(\alpha \mathrm{L}_{1}\right)$ corresponding to infinite $\mathrm{L}_{2}$ or $\mathrm{L}_{3}$. Therefore, higher plateau values are physically inconsistent.

Regarding the estimation of $\mathrm{L}_{1}$, we can observe that using slightly different values for the left plateau level, still lying within the uncertainty in the determination of the plateau, results in a better fitting of the overall profile. Therefore, for each value of $\mathrm{L}_{1}$ one should adjust the plateau to an optimal value and analyze the residual error to determine its suitability (see Sec. II D 5). Instead of estimating each time a suitable plateau value, we choose an interval as large and constant as possible (regardless of the noise) which will be considered as the best plateau estimation. This interval of confidence will allow the computer to finely tune the plateau on a least square sense.

Indeed, if we discard the influence of an offset current as well as the measurement noise and assume the fit to be perfect, the theoretical profile should be proportional to the measurement profile with an unknown scaling factor $A$ that is estimated in the least square sense on the interval of confidence. The estimated $\mathrm{A}$ is then used to rescale the whole profile.

The observed range of $\mathrm{S}_{\mathrm{eff}}$ is four orders of magnitude while the range of diffusion lengths physically relevant for a defined material is less than one order of magnitude. Thus we decided to fit the logarithm of the $S_{\text {eff }}$ rather than $S_{\text {eff }}$ itself to allow a comparable change in the shape of the curves varying the fitting parameters of comparable relative values.

\section{Procedure}

\section{LBIC measurement}

The LBIC system in this investigation uses four modulated lasers (at wavelength $\lambda=635,833,910$, and $980 \mathrm{~nm}$ ) and allows a sharp focusing of the lasers $(\sigma<8 \mu \mathrm{m})$ as well as a step size as small as $1 \mu \mathrm{m}$ in $\mathrm{x}$ and $\mathrm{y}$ direction. The system performs a lock-in measurement of the short circuit current as well as reflectance for each wavelength.

For this investigation it is beneficial to use a sharply focused laser to enhance the contrast at the GBs. Indeed, if the beam is broad, a large amount of carriers would still be collected when the beam is in the surroundings of the GB and would result in a broadening of the dip in the profile and prevent the detailed observation of small features. Another consequence would be a decrease in the relative amplitude of the dip that will decrease the accuracy of the determination of $\mathrm{S}_{\mathrm{eff}}$.

Another mandatory condition is a high spatial resolution, meaning a small step size $(<5 \mu \mathrm{m})$. A large number of points in a region guarantees that even small features will be captured as well as better evaluation of the noise in order to reduce its influence on the fit.

Because of the long duration of a high resolution measurement, a map at low resolution is taken on which regions of interest are determined followed by high resolution mea- 


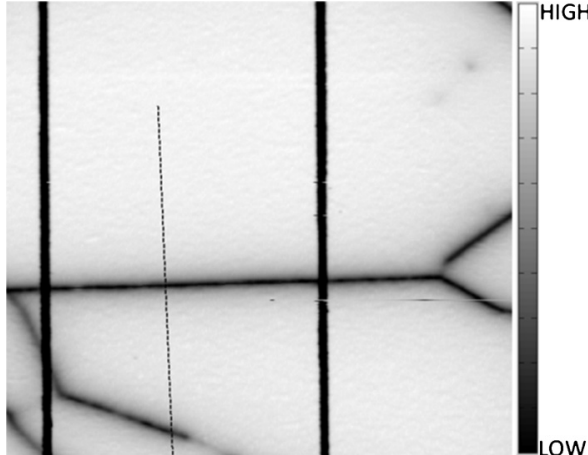

FIG. 2. High resolution LBIC map of the short circuit current at $\lambda$ $=833 \mathrm{~nm}$ with a step size of $2 \mu \mathrm{m}$. The dashed line indicates the linescan extracted from this map whereas the two bold vertical lines belong to the metallization grid.

surements of these regions (see Fig. 2). For determination of a suited area for evaluation, care has to be taken that the assumed invariance in y-direction is given to the highest degree possible.

\section{Profile extraction}

For the selection of a suitable GB profile, one has to consider that the investigated GB is relatively straight and relatively far from other differently oriented GBs in order to fulfill the assumption of invariance along the $y$-axis (see Sec. II A). At least one of the neighboring grains should be larger and longer than $4 \mathrm{~L}_{\text {diff }}$ in order to extract the plateau value.

The linescan is then extracted perpendicular to the GB, the plateau value is determined and the profile is normalized.

The laser beam radius is evaluated by fitting linescans perpendicular to the busbar or fingers of the solar cell front side metallization, providing that they are broader than three times the beam radius. If the beam profile is Gaussian, this profile can be fitted automatically using a scaled complementary error function as a model.

\section{Profile fitting}

As a general rule, the various parameters of the fit influence different parts of the profile. Starting from the outermost part of the profile (the plateau), the first parameter that has an influence on the shape is the diffusion length. Indeed,

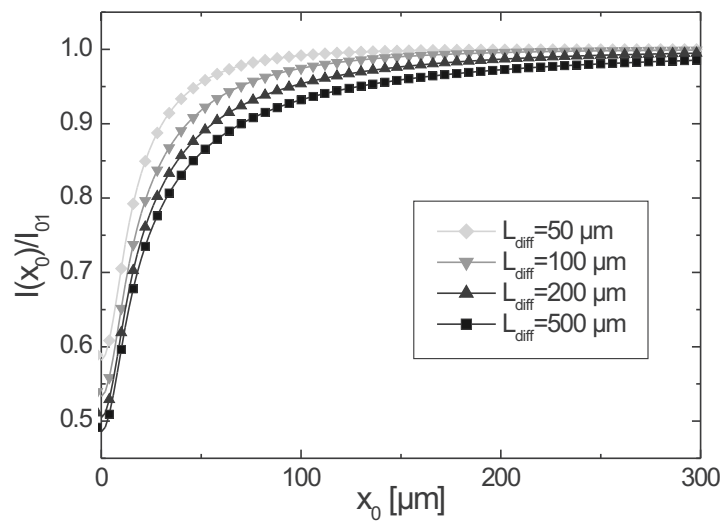

FIG. 3. Simulation of GB LBIC profiles at $\lambda=833 \mathrm{~nm}$ assuming $\sigma$ $=8 \mu \mathrm{m}$ and $\mathrm{S}_{\text {eff }}=10^{5} \mathrm{~cm} / \mathrm{s}$ for $\mathrm{L}_{\text {diff }}=50,100,200$, and $500 \mu \mathrm{m}$.

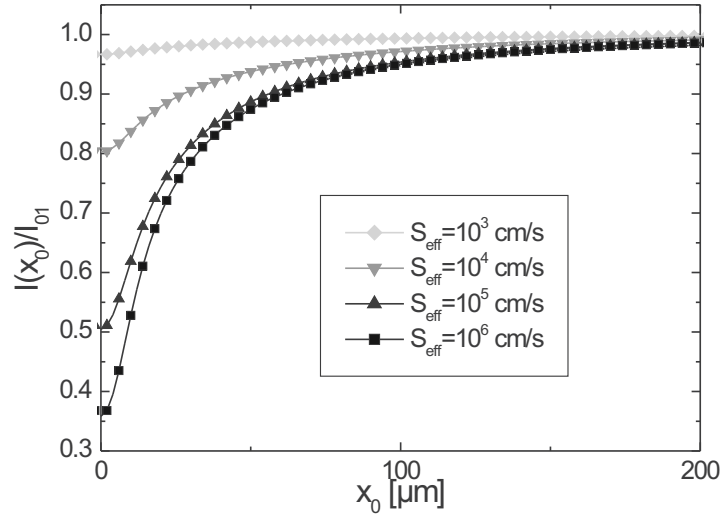

FIG. 4. Simulation of GB profiles at $\lambda=833 \mathrm{~nm}$ assuming $\sigma=8 \mu \mathrm{m}$ and $\mathrm{L}_{\text {diff }}=200 \mu \mathrm{m}$ for $\mathrm{S}_{\mathrm{eff}}=10^{3}, 10^{4}, 10^{5}$, and $10^{6} \mathrm{~cm} / \mathrm{s}$.

a long diffusion length implies that a non negligible fraction of carriers generated relative far from the GB will diffuse toward and reach the GB where they can recombine. The current collected at the junction will therefore smoothly decrease while the laser gets closer to the GB. Thus mainly the outer region of the profile is influenced by $\mathrm{L}_{\mathrm{diff}}$ as shown in Fig. 3.

In the inner part of the dip, $S_{\text {eff }}$ influences mainly the slope of the wall of the dip and its depth as can be observed in Fig. 4.

Finally, due to a more or less extended generation around the GB, the laser beam radius is a parameter that influences directly the depth of the dip, and should therefore be well determined in order to correctly determine $S_{\text {eff }}$. As we can see from Fig. 5, $\sigma$ influences the depth, but to the contrary of $S_{\text {eff }}$, it influences very little the slope of the wall of the dip. A discrepancy on the walls while the depth is correctly reproduced is therefore an indication of an incorrectly estimated $S_{\text {eff }}$ and, in this particular case, an incorrectly estimated $\sigma$.

We can summarize the various zones of influence of each parameter while all the others are kept constant by the schematic of Fig. 6.

The zone in which the most important discrepancy occurs is therefore an indication of the parameter that should be readjusted in a trial and error method. The area close to the GB is the area of highest sensitivity toward errors.

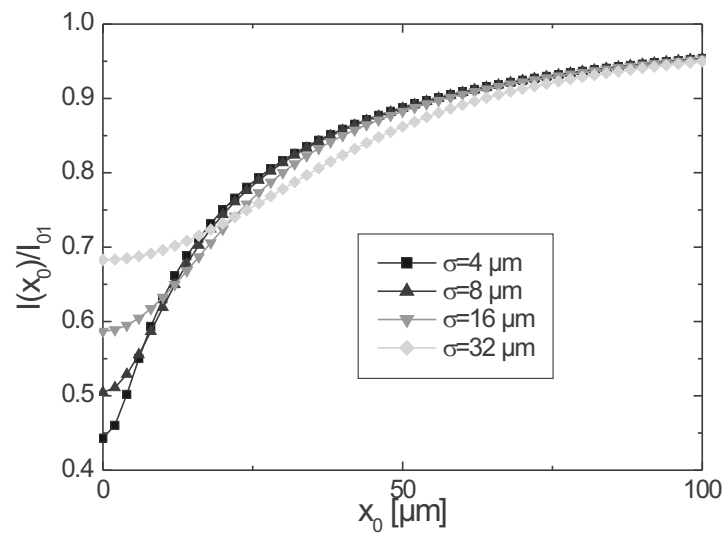

FIG. 5. Simulation of GB profiles at $\lambda=833 \mathrm{~nm}$ assuming $\mathrm{S}_{\text {eff }}=10^{5} \mathrm{~cm} / \mathrm{s}$ and $\mathrm{L}_{\mathrm{diff}}=200 \mu \mathrm{m}$ for $\sigma=4,8,16$, and $32 \mu \mathrm{m}$. 


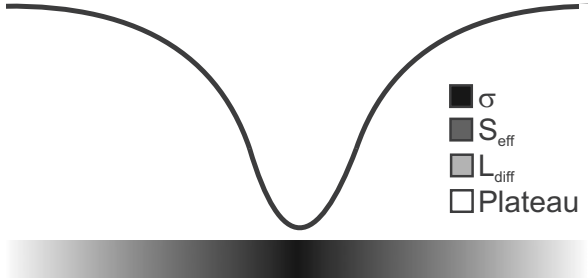

FIG. 6. Zone of influence of each fitting parameter when all the others are set constant.

We suggest to perform a rough fit by starting from the innermost part of the dips. Having set $\sigma$, one then selects appropriate $\mathrm{S}_{\mathrm{eff}} \mathrm{S}$ to match the depth of the dips. However, one needs to adjust $\mathrm{L}_{1}$ and $\mathrm{P}_{2,3}$ to match the shape (see Fig. 3 ), which will have a weak influence on the depth of the dips as one can see in Fig. 4. Thus one needs to readjust the $S_{\text {eff }} \mathrm{s}$ after adjusting the diffusions lengths. This procedure usually reduces the residual error to rather low values, however, one can observe that values of diffusions lengths have an uncertainty of $20 \%-100 \%$ without drastically changing the resulting RMS error. In Sec. II D 5, we propose a more advanced residual error analysis in order to increase the accuracy of the fitted parameters.

\section{Simultaneous fitting of profiles based on different laser wavelengths for charge carrier generation}

An inherent problem with the diffusion length evaluation by this method occurs when the diffusion length is large because of the collection efficiency reaching unity. ${ }^{8}$

Long wavelength light, by penetrating deeper into the wafer, results in a more accurate determination of large diffusion lengths. We, however, face in this case that the dip becomes broader, which shrinks the dimensions of the plateaus and increases the probability of not finding any plateau value to accurately scale the profile. Another limitation is the fact that we get more influence of the back side of the cell on the profile.

We thought therefore of fitting a short wavelength profile, in which the plateau but not the fitted parameters $\left(\mathrm{L}_{1,2,3}\right.$ and $\mathrm{S}_{\mathrm{eff} 1,2}$ ) are accurately determined due to a reduced contrast and reduced lateral extension of the profile, simultaneously with a longer wavelength profile, in which we have the opposite situation. The successful fitting of both profiles gives therefore more reliable parameters than the fitting of only one profile alone. This method could be extended to three different wavelengths of the LBIC system, further enhancing its reliability and accuracy. One has, however, to take care that the penetration depth is in any case much smaller than the wafer thickness. If the residual error is significantly larger for one wavelength than for the other despite of using the same set of parameters, a possible explanation is that the wafer is not homogeneous in depth. The kind of discrepancy encountered can bring some information about the changing wafer quality with depth in the linescan region. For our two lasers at $\lambda=833 \mathrm{~nm}$ and $\lambda=910 \mathrm{~nm}$ we set, respectively, the absorption coefficient to $\alpha_{833}=65 \mathrm{~mm}^{-1}$ and $\alpha_{910}=27 \mathrm{~mm}^{-1} .10$

\section{Residual error analysis}

After performing a rough adjustment of the profile parameters as described in Sec. II D 3, the residual error is the sum of several weak sources of errors that are hardly separable because they are of the same amplitude. They can be, however, more easily discriminated regarding the position at which they occur in the linescan and the range on which they span:

Short range correlated $(1-10 \mu \mathrm{m})$

-LBIC measurement errors and local inhomogeneities in the reflection (see Sec. IV F).

Medium range correlated $(30-100 \mu \mathrm{m})$

-Tilted GB (see Sec. IV C).

-Material inhomogeneities.

Long range correlated $(100-300 \mu \mathrm{m})$

-Back side effect if thickness $\mathrm{t}<\mathrm{L}_{\text {diff }}$ (see Sec. IV D).

$-\mathrm{L}_{\text {diff }}$ noncorrectly adjusted.

Whole profile correlated

-Plateau level not correctly adjusted (interval of confidence not suitably adjusted).

To evaluate the residual regarding these aspects we propose to use the normalized autocorrelation function in conjunction with the residual itself on both laser profiles with different wavelengths.

The influence of a homogeneous back side, which was not taken into account in the model described in Secs. II A and II B, will be symmetric on both sides of the dips. In consequence, this allows to determine one $\mathrm{L}_{\text {diff }}$ value that fits correctly the profile closer to the plateau and another $\mathrm{L}_{\text {diff }}$ value that fits the profile closer to the GB. This phenomenon is characteristic of a back side influence and according to some simulations it seems that in a majority of situations $\mathrm{L}_{\text {diff }}$ is more correctly estimated when fitting closer to the GB.

From the preceding list, asymmetries in the residual can be attributed to lateral inhomogeneities in $\mathrm{L}_{\text {diff }}$ or to tilted GBs. In the case of a tilted GB, however, the asymmetry has a peculiar shape (see Sec. IV C) that could be distinguished from material inhomogeneities if the $\mathrm{GB}$ is tilted by more than $20^{\circ}$.

This residual error analysis allows analyzing more clearly the origin of the deviations from the model prediction and refining the parameters thus improving the overall fit accuracy. This method is proposed as an alternative to the fit of the Fourier transform of the profile introduced by Mittiga and Capizzi. ${ }^{11}$

\section{SPECIAL CASE STUDIES}

The present model can overcome some of the restrictions of former models ${ }^{5-8,12,13}$ providing that model parameters are adapted to the specific case as described in Table I.

\section{A. Scenario 1: "One $L_{\text {diff" }}$}

\section{High $S_{\text {eff }}$}

We extract the profile of a highly active isolated GB from the LBIC maps of the two lasers at $\lambda=833 \mathrm{~nm}$ and $\lambda$ 
TABLE I. Overview of the different scenarios for fitting $\mathrm{L}_{\text {diff }}$ and $\mathrm{s}$ values and their labeling.

\begin{tabular}{|c|c|c|c|c|c|c|}
\hline No. & & $\mathrm{L}_{1}$ & $\mathrm{~s}_{1}$ & $\mathrm{~L}_{2}$ & $\mathrm{~s}_{2}$ & $\mathrm{~L}_{3}$ \\
\hline 1 & "One $L_{\text {diff }} "$ & $\mathrm{~L}$ & s & $\mathrm{L}$ & 0 & $\mathrm{~L}$ \\
\hline 2 & "Two $\mathrm{L}_{\text {diff }}$ ", & $\mathrm{L}_{1}$ & s & $\mathrm{L}_{2}$ & 0 & $\mathrm{~L}_{2}$ \\
\hline 3 & "Two $S_{\text {eff }}$ ", & $\mathrm{L}$ & $\mathrm{s}_{1}$ & $\mathrm{~L}$ & $\mathrm{~s}_{2}$ & $\mathrm{~L}$ \\
\hline 4 & "Defect region" & $\mathrm{L}_{1}$ & 0 & $\mathrm{~L}_{\text {def }}$ & 0 & $\mathrm{~L}_{2}$ \\
\hline $2+3$ & "General model" & $\mathrm{L}_{1}$ & $\mathrm{~s}_{1}$ & $\mathrm{~L}_{2}$ & $\mathrm{~s}_{2}$ & $\mathrm{~L}_{3}$ \\
\hline
\end{tabular}

${ }^{\mathrm{a}}$ Reference 5 .

${ }^{\mathrm{b}}$ References 6 and 12.

${ }^{\mathrm{c}}$ References 7 and 13.

$=910 \mathrm{~nm}$. After normalization the simultaneous fitting is shown in Fig. 7 with a very good agreement between the fit and the measurements.

We are talking of high $\mathrm{S}_{\text {eff }}$ when the normalized depth of the dip, the GB contrast, is more than 0.5, corresponding generally to an $\mathrm{S}_{\text {eff }}$ above $10^{5} \mathrm{~cm} / \mathrm{s}$ for a sharply focused laser $(\sigma<10 \mu \mathrm{m})$.

A high $\mathrm{S}_{\mathrm{eff}} \mathrm{GB}$ presents two attractive features:

(1) The recombination activity is so high that local diffusion length inhomogeneities due to defect clusters or precipitates in the vicinity of the GB can be discarded.

(2) If $L_{\text {diff }}$ differs from either side of the $G B$, a high $S_{\text {eff }}$ allows studying both sides of the dip independently due to a negligible carrier transfer (see Sec. III B). ${ }^{8}$

\section{Low $S_{\text {eff }}$}

A low $S_{\text {eff }}$ at the GB, however, is more difficult to fit accurately due the nonvalidity of the two attractive features of the high $\mathrm{S}_{\text {eff }}$ case. The use of several wavelengths improves the accuracy of the estimations. A fit of a GB with low $S_{\text {eff }}$ is presented in Fig. 8 .

\section{B. Scenario 2: "Two $L_{\text {diff" }}$ and carrier transfer}

The "two $\mathrm{L}_{\text {diff" }}$ " scenario leads to the phenomenon of carrier transfer between both sides of the GB. To demonstrate it, we need to express the equation obtained for this simple case in the form of the sum of three terms each having a physical meaning

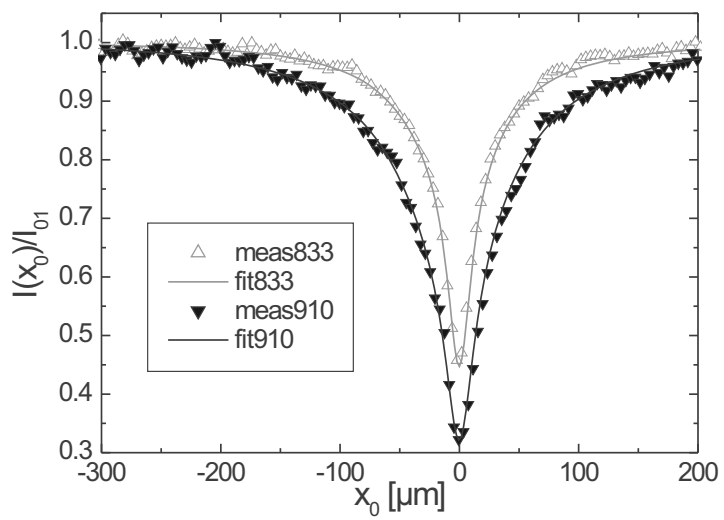

FIG. 7. Simultaneous fitting of a GB presenting a high $S_{\text {eff }}$ at $\lambda=833$ and $910 \mathrm{~nm}$ with $\mathrm{S}_{\text {eff }}=1.4 \times 10^{5} \mathrm{~cm} / \mathrm{s}$ and $\mathrm{L}_{1}=\mathrm{L}_{2}=180 \mu \mathrm{m}$.

$$
\frac{\mathrm{I}\left(\mathrm{x}_{0}\right)}{\mathrm{I}_{01}}=\mathrm{P}-\mathrm{I}_{1}-\mathrm{I}_{2}
$$

with

$$
\begin{aligned}
\mathrm{P}= & \frac{1}{2}\left[2-\left(\frac{\Lambda_{2}-\Lambda_{1}}{\alpha+\Lambda_{2}}\right) \operatorname{erfc}\left(-\frac{\mathrm{x}_{0}}{\sigma}\right)\right], \\
\mathrm{I}_{1}= & \frac{1}{\pi}\left(\Lambda_{1}+\alpha\right) \int_{0}^{\infty} \frac{\mathrm{k}^{2}}{\mathrm{k}^{2}+\alpha^{2}} \times \frac{1}{\mu_{2} \mu_{1}} \\
& \times\left(\frac{\mu_{2}-\mu_{1}}{\mu_{1}} \times \mathrm{U}_{1}+\frac{\mu_{1}-\mu_{2}}{\mu_{2}} \times \mathrm{U}_{2}\right) \mathrm{dk},
\end{aligned}
$$

$$
\mathrm{I}_{2}=\frac{\mathrm{s}}{\pi}\left(\Lambda_{1}+\alpha\right) \int_{0}^{\infty} \frac{\mathrm{k}^{2}}{\mathrm{k}^{2}+\alpha^{2}} \times \frac{1}{\mu_{2} \mu_{1}} \times \frac{\mathrm{U}_{1}+\mathrm{U}_{2}}{\mu_{1}+\mu_{2}+\mathrm{s}} \mathrm{dk},
$$

in which

$$
\begin{aligned}
\mathrm{U}_{\mathrm{n}}= & \exp \left[\left(\frac{\mu_{\mathrm{n}} \sigma}{2}\right)^{2}+(-1)^{\mathrm{n}+1} \mu_{\mathrm{n}} \mathrm{x}_{0}\right] \\
& \times \operatorname{erfc}\left[\frac{\mu_{\mathrm{n}} \sigma}{2}+(-1)^{\mathrm{n}+1} \frac{\mathrm{x}_{0}}{\sigma}\right] .
\end{aligned}
$$

$\mathrm{P}$ is the normalized plateau level corresponding to the current obtained without GB, considering a purely one-dimensional model.

We observe that $\mathrm{I}_{1}$ is independent of $\mathrm{s}$, and therefore of the GB, and cancels out if $\mu_{1}=\mu_{2}$ implying $\mathrm{L}_{1}=\mathrm{L}_{2}$. There-

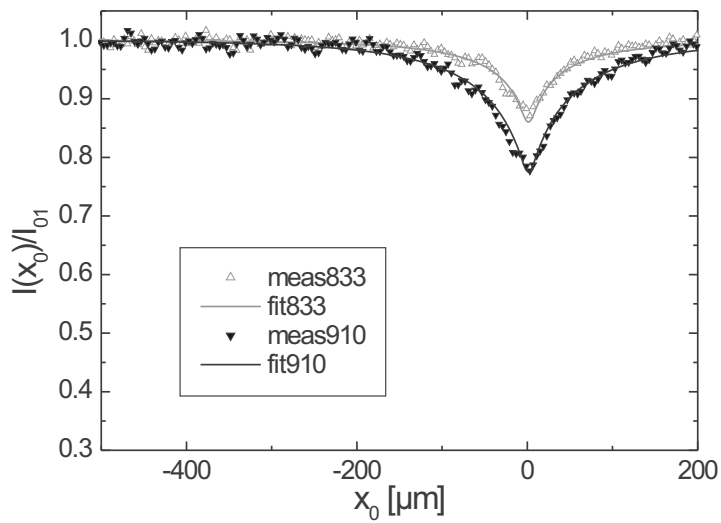

FIG. 8. Simultaneous fitting of a GB presenting a low $S_{\text {eff }}$ at $\lambda=833$ and 910 $\mathrm{nm}$ with $\mathrm{S}_{\mathrm{eff}}=5.6 \times 10^{3} \mathrm{~cm} / \mathrm{s}$ and $\mathrm{L}_{1}=\mathrm{L}_{2}=200 \mu \mathrm{m}$. 


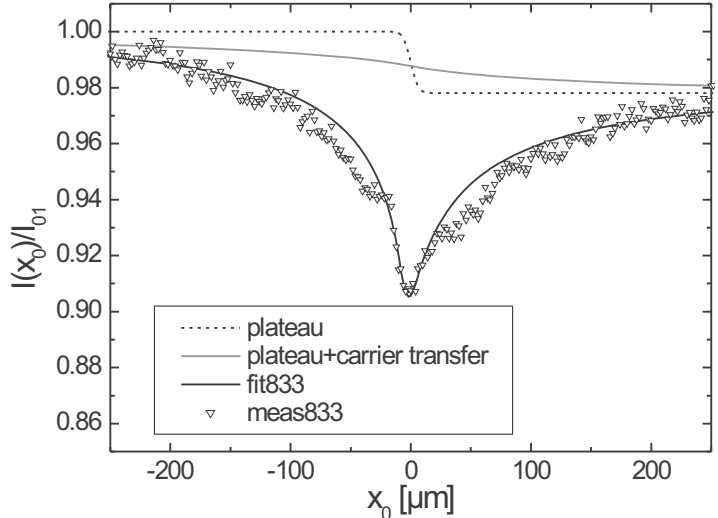

FIG. 9. Fitting of a "two $L_{\text {diff" }}$ " situation at $\lambda=833 \mathrm{~nm}$ with $\mathrm{S}_{\text {eff }}=2.3$ $\times 10^{3} \mathrm{~cm} / \mathrm{s}, \mathrm{L}_{1}=1 \mathrm{~mm}$ and $\mathrm{L}_{2}=340 \mu \mathrm{m}$. The plateau dependence on the position is shown as well as its correction by the carrier transfer term.

fore, this term corresponds to a carrier transfer between the two regions that cannot be taken in account by a onedimensional model.

Finally, we observe that $\mathrm{s}$ is a factor in front of the $\mathrm{I}_{2}$ expression and therefore $I_{2}$ vanishes completely for $s=0$, meaning no GB.

In the case of a low $\mathrm{S}_{\text {eff }} \mathrm{GB}$ with largely differing diffusion lengths on both sides, $\mathrm{I}_{1}$ becomes of the same order of magnitude and even larger than $\mathrm{I}_{2}$. In this case, the minimum of the collected current is no more exactly located at the GB position, and in extreme cases the dip induced by the GB can even fully disappear.

This phenomenon observed by von Roos and Luke ${ }^{6}$ finds here its justification and makes every low $S_{\text {eff }}$ fitting much more critical as high $\mathrm{S}_{\mathrm{eff}}$ ones.

We performed a fit on a profile and show the plateau and the contribution of the carrier transfer term in Fig. 9. We observe that in comparison to the profile of the onedimensional case (plateau) the profile of the two-dimensional case (plateau+carrier transfer) is smoother because of the lateral carrier transfer from one side to the other.

\section{Scenario 3: "Two $\mathrm{S}_{\text {eff }}$}

In this section we present the fitting of the linescan extracted in Fig. 2 in which two GBs are separated by a distance that is too short to allow a study of each one independently (Fig. 10).

In the case where two GBs are located at a distance of less than $4 \mathrm{~L}_{\text {diff }}$ from each other, the region in between is affected by the recombination of both GBs and the maximum current found in this region cannot be considered as a plateau value by definition. Therefore, the problem cannot be decoupled in the inner part even considering highly recombinative GBs. Our model allows therefore studying this situation providing that our profile includes one plateau region on the left side.

\section{Scenario 4: "Defect region"}

On some profiles (see Fig. 11) an accurate fit could be obtained only by assuming a rather large laser beam radius $\sigma>30 \mu \mathrm{m}$. This finding was not in agreement with indepen-

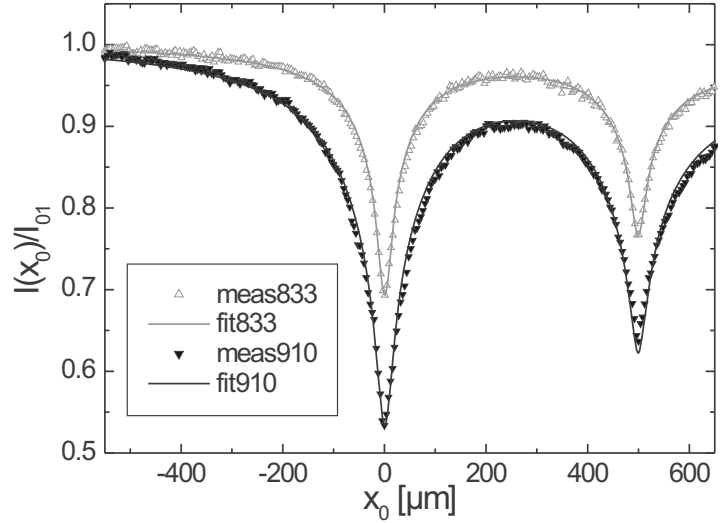

FIG. 10. Simultaneous fitting of two GBs at $\lambda=833$ and $910 \mathrm{~nm}$ with $\mathrm{S}_{\text {eff1 }}=2 \times 10^{4} \mathrm{~cm} / \mathrm{s}, \quad \mathrm{S}_{\text {eff2 }}=1 \times 10^{4} \mathrm{~cm} / \mathrm{s}$ and $\mathrm{L}_{1}=\mathrm{L}_{2}=900 \mu \mathrm{m}$ and $\mathrm{L}_{3}$ $=320 \mu \mathrm{m}$.

dently estimated $\sigma$ values that were below $10 \mu \mathrm{m}$. One possible explanation is the presence of a narrow defective region around the GB. In this case, these defects will lower the diffusion length in the immediate surroundings of the GB which will induce a broadening of the dip profile. If $\mathrm{S}_{\mathrm{eff}}$ of this GB is relatively weak, the recombination of these defects can exceed the recombination of the GB. In this case, a reasonable approximation is to define a narrow region centered around the bottom of the dip with low $\mathrm{L}_{\text {diff. }}$ Even if our model can consider only abrupt transitions of $\mathrm{L}_{\text {diff }}$, a good agreement can be found adapting the width of the region and

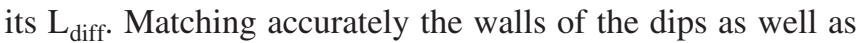
its bottom for a given $\sigma$ requires a defined couple of width and $\mathrm{L}_{\text {diff. }}$ For this approximation the transition between regions of different diffusion lengths does not contain GBs and therefore we set $S_{\text {eff } 1}=S_{\text {eff } 2}=0$.

Figure 11 shows the fitting discrepancy that occurs while fitting accurately the outer curvature $\left(\mathrm{L}_{\text {diff }}\right)$ and the bottom of the dip ( $\mathrm{S}_{\mathrm{eff}}$ and $\sigma$ although $\sigma$ is known). However, considering a defective region of $35 \mu \mathrm{m}$ width with $\mathrm{L}_{\text {diff }}$ $=32 \mu \mathrm{m}$, we observe a much better agreement with the measurement.

\section{E. Depth inhomogeneity}

We observe in Fig. 12 that while fitting accurately the profile for $\lambda=833 \mathrm{~nm}$, the profile for $\lambda=910 \mathrm{~nm}$ is under-

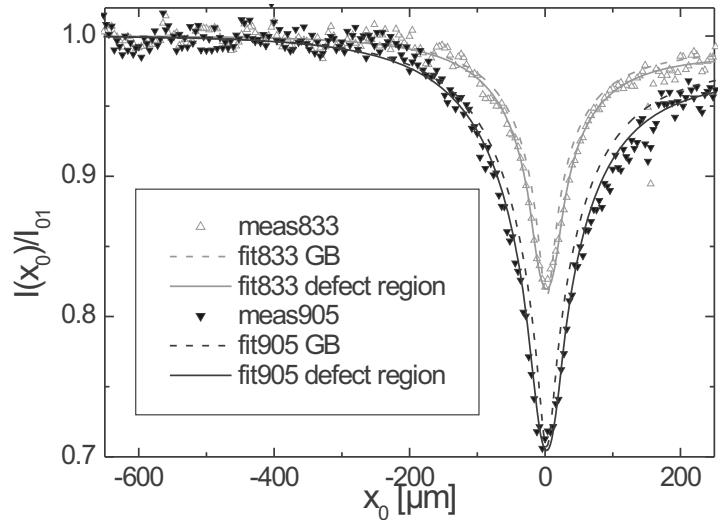

FIG. 11. Simultaneous fitting of a GB at $\lambda=833$ and $910 \mathrm{~nm}$ with $\mathrm{L}_{1}$ $=200 \mu \mathrm{m}, \mathrm{L}_{2}=175 \mu \mathrm{m}$ and assuming the influence of a GB (dashed lines) with $\mathrm{S}_{\text {eff }}=8.8 \times 10^{3} \mathrm{~cm} / \mathrm{s}$ as well as assuming a narrow defective region of $35 \mu \mathrm{m}$ of width and $\mathrm{L}_{\mathrm{def}}=32 \mu \mathrm{m}$ (full line). 


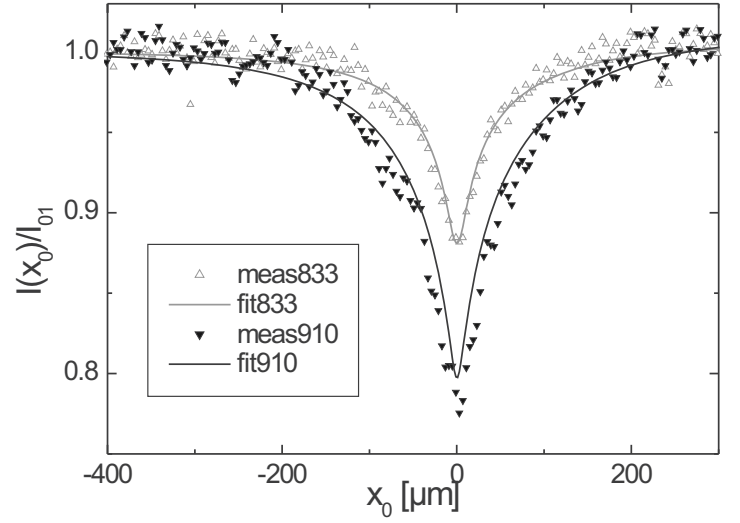

FIG. 12. Simultaneous fitting of a GB at $\lambda=833$ and $910 \mathrm{~nm}$ with $\mathrm{L}_{1}=\mathrm{L}_{2}$ $=200 \mu \mathrm{m}$ and $\mathrm{S}_{\text {eff }}=4.6 \times 10^{3} \mathrm{~cm} / \mathrm{s}$ showing a discrepancy between the two laser fittings which indicates a depth inhomogeneity at the GB.

estimated. This phenomenon could be explained by a more recombinative region in the depth of the bulk that is reachable only by the deeper penetrative laser light at $910 \mathrm{~nm}$. This phenomenon could be explained by a surface gettering or hydrogenation effect reducing $\mathrm{S}_{\mathrm{eff}}$ of the GB at the surface while keeping essentially a higher value in depth. Taking in account in our model this phenomenon would, however, require a much more advanced investigation on the gettering or passivation effect.

\section{DISCUSSION ON THE LIMITATIONS}

\section{A. Long and straight GB necessary}

The strong requirement of the invariance of the problem along the y-axis (see Sec. II A) is a main cause of limited applicability of this theory for the majority of GBs in a relatively small grained material. One of the best materials suited for this model is therefore vertically grown silicon ribbon material like edge-defined film-fed growth (EFG) and String Ribbon ${ }^{14}$ for which the very elongated grains provide this condition. This model can as well be applied to multicrystalline silicon in which the grains are large enough compared to $\mathrm{L}_{\mathrm{diff}}$ to fulfill this condition.

\section{B. One long plateau necessary}

The necessity of normalizing the profile to one plateau value explained in Sec. II B imposes the mandatory condition of a very accurate estimation of a plateau level. A plateau implies being far away from any GB so that one can neglect their influence. Therefore, one grain should be at least $4 \mathrm{~L}_{\text {diff }}$ large. As pointed out by Corkish, ${ }^{8}$ a variation of $1 \%$ of the plateau value can induce an error of $100 \%$ on the diffusion length value when the diffusion length is high.

\section{Tilted GB}

Another reason for asymmetry can be the nonperpendicularity of the GB to the wafer surface. Simulations (Fig. 13) show that for up to $20^{\circ}$ of tilt angle, this influence could be neglected. We observe that this influence is more pronounced with increasing laser wavelength. This phenomenon is due to a higher penetration depth that induces deviations

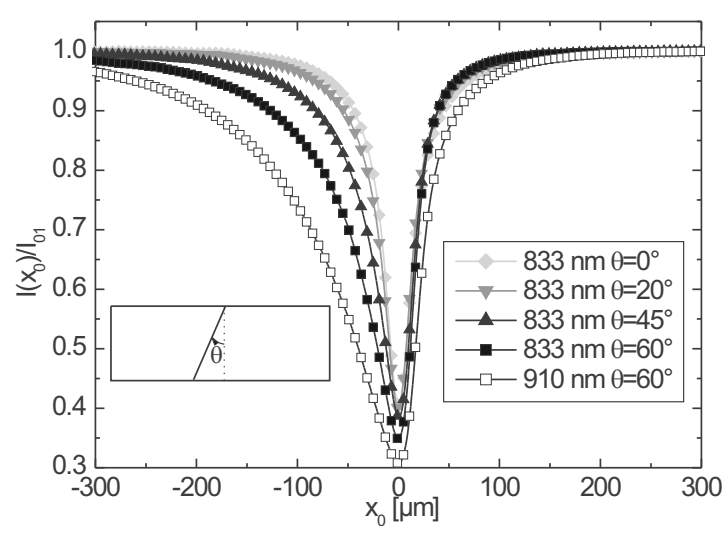

FIG. 13. Simulation of a GB contrast profile when the GB is tilted by an angle $\theta=0^{\circ}, 20^{\circ}, 45^{\circ}$, and $60^{\circ}$ with $\mathrm{S}_{\text {eff }}=10^{6} \mathrm{~cm} / \mathrm{s}$ and $\mathrm{L}_{\text {diff }}=100 \mu \mathrm{m}$ on a $250 \mu \mathrm{m}$ thick structure for $\lambda=910$ and $833 \mathrm{~nm}$.

on a larger distance scale. In the case of an asymmetric profile with the same plateau on both sides (no differing diffusion length) and no close GB, we could deduce that the GB is tilted and is not accurately fitted with any model that does not take into account the tilt angle. Unfortunately, a nonperpendicular GB is a problem that cannot be solved analytically ${ }^{15}$ and thus requires full numerical methods.

\section{Long diffusion length and back side effect}

In the case of high $\mathrm{L}_{\text {diff }}$, the collection efficiency approaches unity and therefore the profile does not change significantly between two very different $\mathrm{L}_{\text {diff }}$ fittings. ${ }^{8}$ Additionally, due to the fact that a non negligible part of the carriers diffuses toward the back side of the cell, the approximation of an infinite thickness of the wafer becomes incorrect, particularly when the penetration depth of the light is large. For our laser wavelengths of 833 and $910 \mathrm{~nm}$ (absorption depths of approximately 15 and $37 \mu \mathrm{m}$ ) we are well below typical wafer thicknesses of around $200 \mu \mathrm{m}$. Therefore, the proposed model can strictly speaking only be applied for cases where the wafer thickness is larger than the laser penetration depth and $\mathrm{L}_{\text {diff. }}$. Only a new model taking the finite thickness into account and therefore adding the back side to the present model can satisfactorily solve this problem.

\section{E. Injection level}

Due to the various phenomena mentioned in the introduction, $\mathrm{S}_{\text {eff }}$ is strongly injection dependent in the intermediate and high injection regime and weakly dependant in the very low injection regime. ${ }^{4}$ We have, therefore, evaluated the maximum injection level to be of the order of $\Delta \mathrm{n} \approx 3$ $\times 10^{13} \mathrm{~cm}^{-3}$ for a large $\mathrm{L}_{\mathrm{diff}}=1 \mathrm{~mm}$ by means of a threedimensional simulation considering our four lasers finely focused to $\sigma=8 \mu \mathrm{m}$ (maximum possible injection with our setup, no bias light). Considering that the wafer doping, and so the majority carrier density, is of the order of $10^{15}-10^{16} \mathrm{~cm}^{-3}$, we are clearly in low injection. According to Oualid, ${ }^{4}$ this injection level is unfortunately in the range where $S_{\text {eff }}$ is still significantly dependant on the injection level. Therefore, the determination of $\mathrm{S}_{\text {eff }}$ presented here has to be compared for the same condition of injection. 


\section{F. Nonconstant reflection over the profile}

Local inhomogeneity of the semiconductor surface can cause small short range variations in the reflection $(\mathrm{R})$ of the order of $1 \%-2 \%$ in the experimental profile. As the plateau value is a very sensitive parameter, a variation in this order could perturb the results of this method. These variations that appear like an increased noise on the profile could in principle be avoided by normalizing the profile by (1-R). Indeed (1-R) appears in the expression of the left plateau level $\mathrm{I}_{01}$ [see Eq. (A29)]. Our LBIC system can measure the reflectance associated with each laser, however, only in a relative manner. The use of integral reflection data measured in an absolute manner by a spectrophotometer can provide a calibration for the LBIC reflection data and would allow performing this normalization procedure. But it should be considered that these variations are probably caused by the varying orientations of the microscopic small features of the semiconductor surface. Therefore, a phenomenon associated with nonperpendicularity of the internal beam, like changes in the generation volume function $g(r)$, cannot be taken into account by this simple procedure.

\section{CONCLUSION}

The limits of the method of extracting values of $\mathrm{L}_{\text {diff }}$ and $\mathrm{S}_{\text {eff }}$ around a GB by using LBIC data pioneered by Donolato ${ }^{5}$ in 1983 and improved by Corkish ${ }^{8}$ in 1998 were pushed further. We developed an advanced model that aimed at fitting various $\mathrm{GB}$ contrast profiles situations. We implemented this model in a MATLAB graphical interface making it a powerful tool for GB investigation. Advances in the determination of the plateau level by means of setting an interval of confidence as well as in the simultaneous fitting of several laser wavelengths, assuming an in depth invariance of the surface recombination velocities and effective diffusion lengths lead to a more accurate determination of the diffusion lengths compared to previous methods. We demonstrated the application of the model to several GB situations and show a very good agreement between measurements and fitted curves. The analysis of the discrepancies between measurement and fitting can reveal the presence of features like in depth inhomogeneities or narrow defective regions that are nearly impossible to access by other measurement and interpretation means. Investigation of the dependence of the effective surface recombination velocities to the injection level, varying the power of the laser and/or the laser beam radius, will allow getting more information about the GB defects in real multicrystalline materials.

\section{ACKNOWLEDGMENTS}

The authors want to thank Axel Herguth and Johannes Junge for providing assistance in the high resolution LBIC maps measurements presented in this work. The authors would like to thank Robert Denk and Philippe Cance for very helpful discussions about the resolution methods of PDEs. Part of this work was funded by the EC in the CrystalClear (Grant No. SES6-CT-2003-502583) and the German BMU in the SolarFocus (Grant No. 0327650H) project. The content of this publication is the responsibility of the authors.
TABLE II. List of the boundaries in the $\mathrm{x}$-axis.

\begin{tabular}{lcccc}
\hline \hline $\mathrm{m}$ & 0 & 1 & 2 & 3 \\
\hline $\mathrm{x}_{\mathrm{m}}$ & $-\infty$ & 0 & $\mathrm{w}$ & $+\infty$ \\
\hline \hline
\end{tabular}

\section{APPENDIX A: MODEL DERIVATION}

After reducing the problem to two dimensions $(\mathrm{x}, \mathrm{z})$, it is convenient to consider separately the diffusion problem in the three regions in which the semiconductor is divided by the two GBs. We define therefore the respective $\mathrm{x}$ boundaries in Table II.

We then introduce the restriction $G_{i, j}$ corresponding to a two-dimensional Green function for region $\mathrm{i}$ when the point source is located in region $\mathrm{j}$ at $\left(\mathrm{x}_{\mathrm{ps}}, \mathrm{z}_{\mathrm{ps}}\right)$. Thus, the problem can be expressed as the following system of coupled PDEs:

$$
\frac{\partial^{2} \mathrm{G}_{\mathrm{i}, \mathrm{j}}}{\partial \mathrm{x}^{2}}+\frac{\partial^{2} \mathrm{G}_{\mathrm{i}, \mathrm{j}}}{\partial \mathrm{z}^{2}}-\Lambda_{\mathrm{i}}^{2} \mathrm{G}_{\mathrm{i}, \mathrm{j}}=-\delta_{\mathrm{ij}} \frac{1}{\mathrm{D}_{\mathrm{p}}} \delta\left(\mathrm{x}-\mathrm{x}_{\mathrm{ps}}\right) \delta\left(\mathrm{x}-\mathrm{z}_{\mathrm{ps}}\right)
$$

with $\Lambda_{\mathrm{i}}=1 / \mathrm{L}_{\mathrm{i}}, \mathrm{i}=1,2,3, \mathrm{j}=1,2,3$, and $\delta_{\mathrm{ij}}$ the Kronecker delta symbol.

It can be checked that the boundary conditions of Eqs. (3a)-(3d) become, respectively,

$$
\begin{aligned}
& \left.\mathrm{G}_{1, \mathrm{j}}\right|_{\mathrm{x}=0}=\left.\mathrm{G}_{2, \mathrm{j}}\right|_{\mathrm{x}=0} \\
& \left.\mathrm{G}_{2, \mathrm{j}}\right|_{\mathrm{x}=\mathrm{w}}=\left.\mathrm{G}_{3, \mathrm{j}}\right|_{\mathrm{x}=\mathrm{w}} \\
& \left.\frac{\partial \mathrm{G}_{2, \mathrm{j}}}{\partial \mathrm{x}}\right|_{\mathrm{x}=0}-\left.\frac{\partial \mathrm{G}_{1, \mathrm{j}}}{\partial \mathrm{x}}\right|_{\mathrm{x}=0}=\left.\mathrm{s}_{1} \mathrm{G}_{1, \mathrm{j}}\right|_{\mathrm{x}=0} \\
& \left.\frac{\partial \mathrm{G}_{3, \mathrm{j}}}{\partial \mathrm{x}}\right|_{\mathrm{x}=\mathrm{w}}-\left.\frac{\partial \mathrm{G}_{2, \mathrm{j}}}{\partial \mathrm{x}}\right|_{\mathrm{x}=\mathrm{w}}=\left.\mathrm{s}_{2} \mathrm{G}_{2, \mathrm{j}}\right|_{\mathrm{x}=\mathrm{w}}
\end{aligned}
$$

and Eq. (2) becomes

$$
\left.\mathrm{G}_{\mathrm{i}, \mathrm{j}}\right|_{\mathrm{z}=0}=0 \text {. }
$$

In this representation, the solution for any arbitrary $g(r)$ is a convolution product of the point source carrier collection probability $\mathrm{Q}$ by $\mathrm{h}$, the projection in the $\mathrm{x}, \mathrm{z}$-plane of the volume generation function $\mathrm{g}(\mathrm{r})$ induced by an electron/laser beam centered at $\mathrm{x}_{0}$

$$
\mathrm{I}\left(\mathrm{x}_{0}\right)=\mathrm{q} \int_{-\infty}^{\infty} \int_{0}^{\infty} \mathrm{Q}\left(\mathrm{x}_{\mathrm{ps}}, \mathrm{z}_{\mathrm{ps}}\right) \times \mathrm{h}\left(\mathrm{x}_{\mathrm{ps}}-\mathrm{x}_{0}, \mathrm{z}_{\mathrm{ps}}\right) \mathrm{dz} \mathrm{pss}_{\mathrm{ps}} \mathrm{dx}_{\mathrm{ps}}
$$

$$
\mathrm{h}(\mathrm{x}, \mathrm{z})=\int_{-\infty}^{\infty} \mathrm{g}(\mathrm{x}, \mathrm{y}, \mathrm{z}) \mathrm{dy}
$$

We need here to introduce the point source carrier collection probability when the point source is located in region $\mathrm{j}$, named $\mathrm{Q}_{\mathrm{j}}$, that is expressed by making use of Eq. (7) and adding the current contribution of each region 


$$
\mathrm{Q}_{\mathrm{j}}\left(\mathrm{x}_{\mathrm{ps}}, \mathrm{z}_{\mathrm{ps}}\right)=\left.\mathrm{D}_{\mathrm{p}} \sum_{\mathrm{i}=1}^{3} \int_{\mathrm{x}_{\mathrm{i}-1}}^{\mathrm{x}_{\mathrm{i}}} \frac{\partial \mathrm{G}_{\mathrm{i}, \mathrm{j}}}{\partial \mathrm{z}}\right|_{\mathrm{z}=0} \mathrm{dx} .
$$

The total current generated for a nondiscrete source centered at $\mathrm{x}_{0}$ can then be considered as the sum of the contribution of all the points of this source and therefore involves $\mathrm{Q}_{1,2,3}$ when the source has points in the three regions of the semiconductor. Therefore, Eq. (A4) becomes

$$
\begin{aligned}
\mathrm{I}\left(\mathrm{x}_{0}\right)= & \mathrm{q} \int_{0}^{\infty} \sum_{\mathrm{j}=1}^{3} \int_{\mathrm{x}_{\mathrm{j}-1}}^{\mathrm{x}_{\mathrm{j}}} \mathrm{Q}_{\mathrm{j}}\left(\mathrm{x}_{\mathrm{ps}}, \mathrm{z}_{\mathrm{ps}}\right) \times \mathrm{h}\left(\mathrm{x}_{\mathrm{ps}}\right. \\
& \left.-\mathrm{x}_{0}, \mathrm{z}_{\mathrm{ps}}\right) \mathrm{dx}_{\mathrm{ps}} \mathrm{d} \mathrm{z}_{\mathrm{ps}} .
\end{aligned}
$$

Using the sine Fourier transform that respects the boundary condition of Eq. (A3)

$$
\mathrm{G}_{\mathrm{i}, \mathrm{j}}(\mathrm{x}, \mathrm{z})=\int_{0}^{\infty} \mathrm{b}_{\mathrm{i}, \mathrm{j}}(\mathrm{x}, \mathrm{k}) \sin (\mathrm{zk}) \mathrm{dk}
$$

and making use of the identity

$$
\delta\left(\mathrm{z}-\mathrm{z}_{\mathrm{ps}}\right)=\frac{2}{\pi} \int_{0}^{\infty} \sin \left(\mathrm{z}_{\mathrm{ps}} \mathrm{k}\right) \sin (\mathrm{zk}) \mathrm{dk} .
$$

Equation (A1) reduces to an ordinary differential equation (ODE) in $\mathrm{x}$

$$
\frac{\partial^{2} \mathrm{~b}_{\mathrm{i}, \mathrm{j}}}{\partial \mathrm{x}^{2}}-\mu_{i}^{2} \mathrm{~b}_{\mathrm{i}, \mathrm{j}}=-\delta_{\mathrm{i}, \mathrm{j}} \frac{2}{\pi \mathrm{D}_{\mathrm{p}}} \delta\left(\mathrm{x}-\mathrm{x}_{\mathrm{ps}}\right) \sin \left(\mathrm{z}_{\mathrm{ps}} \mathrm{k}\right)
$$

with $\mu_{\mathrm{i}}=\left(\mathrm{L}_{\mathrm{i}}^{-2}+\mathrm{k}^{2}\right)^{1 / 2}=\left(\Lambda_{\mathrm{i}}^{2}+\mathrm{k}^{2}\right)^{1 / 2}$.

Because the boundary conditions of Eqs. (A2a)-(A2d) involve only the $\mathrm{x}$-variable and the sine Fourier transform affects only the $z$-variable, these equations remain unchanged through this transformation and the boundary conditions of these ODEs are Eqs. (A2a)-(A2d) replacing $\mathrm{G}_{\mathrm{i}, \mathrm{j}}$ by $b_{i, j}$.

Because we are dealing with an unbounded spatial variable (x) and therefore need a bilateral transformation, these ODEs are solved most adequately by using the Fourier transform.

The general solutions of Eq. (A10) are therefore

$$
\mathrm{b}_{\mathrm{i}, \mathrm{j}}=\mathrm{A}_{\mathrm{i}, \mathrm{j}} \mathrm{e}^{\mu_{\mathrm{i}} \mathrm{x}}+\mathrm{B}_{\mathrm{i}, \mathrm{j}} \mathrm{e}^{-\mu_{\mathrm{i}} \mathrm{x}}+\delta_{\mathrm{i}, \mathrm{j}} \frac{1}{\pi \mathrm{D}_{\mathrm{p}} \mu_{\mathrm{i}}} \sin \left(\mathrm{z}_{\mathrm{ps}} \mathrm{k}\right) \mathrm{e}^{-\mu_{\mathrm{i}}\left|\mathrm{x}-\mathrm{x}_{\mathrm{ps}}\right|}
$$

in which $\mathrm{A}_{\mathrm{i}, \mathrm{j}}$ and $\mathrm{B}_{\mathrm{i}, \mathrm{j}}$ are some constants which have to be determined by using the boundary conditions Eqs. (A2a)-(A2d) considering $b_{i, j}$ instead of $G_{i, j}$. For convergence reasons at infinity $B_{1, j}$ and $A_{3, j}$ have to be 0 .

The absolute value in Eq. (A11) can be treated by taking into account the position of the considered GB $\left(\mathrm{x}_{\mathrm{b}}=0\right.$ for GB1 and $x_{b}=w$ for GB2) and changing the exponential expression sign if $\left(\mathrm{x}_{\mathrm{b}}-\mathrm{x}_{\mathrm{ps}}\right)<0$.

For $\mathrm{j}=1$ inserting Eq. (A11) in Eqs. (A2a)-(A2d) leads, respectively, to the following system in matrix form:

$$
\begin{aligned}
& {\left[\begin{array}{cccc}
-\mu_{1} & \mu_{1} & \mu_{1} & 0 \\
\mu_{1} & \mu_{2}+\mathrm{s}_{1} & -\mu_{2}+\mathrm{s}_{1} & 0 \\
0 & \mathrm{e}^{-\mu_{2} \mathrm{w}} & \mathrm{e}^{\mu_{2} \mathrm{w}} & -\mathrm{e}^{-\mu_{3} \mathrm{w}} \\
0 & -\mu_{2} \mathrm{e}^{-\mu_{2} \mathrm{w}} & \mu_{2} \mathrm{e}^{\mu_{2} \mathrm{w}} & \left(\mu_{3}+\mathrm{s}_{2}\right) \mathrm{e}^{-\mu_{3} \mathrm{w}}
\end{array}\right] \times\left[\begin{array}{c}
\mathrm{A}_{1,1} \\
\mathrm{~A}_{2,1} \\
\mathrm{~B}_{2,1} \\
\mathrm{~B}_{3,1}
\end{array}\right]} \\
& =\left[\begin{array}{c}
\mathrm{Ce}^{\mu_{1} \mathrm{x}_{\mathrm{ps}}} \\
\mathrm{Ce}^{\mu_{1} \mathrm{x}_{\mathrm{ps}}} \\
0 \\
0
\end{array}\right]
\end{aligned}
$$

with

$$
\mathrm{C}=\frac{1}{\pi \mathrm{D}_{\mathrm{p}}} \sin \left(\mathrm{z}_{\mathrm{ps}} \mathrm{k}\right) .
$$

Using Eq. (A6) and Eq. (A8), $\mathrm{Q}_{\mathrm{j}}$ can be expressed as

$$
\mathrm{Q}_{\mathrm{j}}\left(\mathrm{x}_{\mathrm{ps}}, \mathrm{z}_{\mathrm{ps}}\right)=\mathrm{D}_{\mathrm{p}} \int_{0}^{\infty}\left[\sum_{\mathrm{i}=1}^{3} \int_{\mathrm{x}_{\mathrm{i}-1}}^{\mathrm{x}_{\mathrm{i}}} \mathrm{b}_{\mathrm{i}, \mathrm{j}}(\mathrm{x}, \mathrm{k}) \mathrm{dx}\right] \mathrm{k} \mathrm{dk} .
$$

For $\mathrm{j}=1$ using Eq. (A11) and taking the absolute value into account by separating the range of integration

$$
\begin{aligned}
\mathrm{Q}_{1}\left(\mathrm{x}_{\mathrm{ps}}, \mathrm{z}_{\mathrm{ps}}\right)= & \mathrm{D}_{\mathrm{p}} \int_{0}^{\infty}\left[\frac{\mathrm{A}_{1,1}}{\mu_{1}}-\frac{\mathrm{C}}{\mu_{1}^{2}}\left(2-\mathrm{e}^{\mu_{1} \mathrm{x}_{\mathrm{ps}}}\right)\right. \\
& +\frac{\mathrm{B}_{2,1}+\mathrm{A}_{2,1} \mathrm{e}^{\mu_{2} \mathrm{w}}}{\mu_{2}}\left(1-\mathrm{e}^{-\mu_{2} \mathrm{w}}\right) \\
& \left.+\frac{\mathrm{B}_{3,1}}{\mu_{3}} \mathrm{e}^{-\mu_{3} \mathrm{w}}\right] \mathrm{k} \mathrm{dk} .
\end{aligned}
$$

The tedious system of Eqs. (A12) is solved and the constants replaced in Eq. (A15) by use of the MATHEMATICA software package ${ }^{16}$ leading to an expression of the form

$$
\mathrm{Q}_{1}\left(\mathrm{x}_{\mathrm{ps}}, \mathrm{z}_{\mathrm{ps}}\right)=\mathrm{J}_{1}\left(\mathrm{z}_{\mathrm{ps}}\right)-\mathrm{Q}_{1}^{*}\left(\mathrm{x}_{\mathrm{ps}}, \mathrm{z}_{\mathrm{ps}}\right)
$$

with

$$
\mathrm{J}_{\mathrm{j}}\left(\mathrm{z}_{\mathrm{ps}}\right)=\frac{2}{\pi} \int_{0}^{\infty} \frac{\mathrm{k}}{\Lambda_{\mathrm{j}}^{2}+\mathrm{k}^{2}} \sin \left(\mathrm{z}_{\mathrm{ps}} \mathrm{k}\right) \mathrm{dk}=\mathrm{e}^{-\Lambda_{\mathrm{j}} \mathrm{z}_{\mathrm{ps}}}
$$

and

$$
\mathrm{Q}_{1}^{*}\left(\mathrm{x}_{\mathrm{ps}}, \mathrm{z}_{\mathrm{ps}}\right)=\frac{2}{\pi} \int_{0}^{\infty} \mathrm{f}_{1}(\mathrm{k}) \times \mathrm{e}^{\mu_{1} \mathrm{x}_{\mathrm{ps}}} \sin \left(\mathrm{z}_{\mathrm{ps}} \mathrm{k}\right) \mathrm{dk}
$$

and

$$
\begin{aligned}
\mathrm{f}_{1}(\mathrm{k})= & \frac{\mathrm{k}}{\mu_{1}} \frac{1}{\mathrm{D}(\mathrm{k})}\left\{\mathrm{e}^{2 \mu_{2} \mathrm{w}} \mu_{3}\left(\mu_{2}+\mu_{3}+\mathrm{s}_{2}\right)\left[\mu_{2}\left(\mu_{2}+\mathrm{s}_{1}\right)-\mu_{1}^{2}\right]\right. \\
& +2 \mathrm{e}^{\mu_{2} \mathrm{w}} \mu_{1}^{2}\left[\mu_{3}\left(\mu_{3}+\mathrm{s}_{2}\right)-\mu_{2}^{2}\right]-\mu_{3}\left(\mu_{3}-\mu_{2}+\mathrm{s}_{2}\right) \\
& \left.\times\left[\mu_{2}\left(\mathrm{~s}_{1}-\mu_{2}\right)+\mu_{1}^{2}\right]\right\}
\end{aligned}
$$

and

$$
\begin{aligned}
\mathrm{D}(\mathrm{k})= & \mu_{1} \mu_{2} \mu_{3}\left[\mathrm{e}^{2 \mu_{2} \mathrm{w}}\left(\mu_{1}+\mu_{2}+\mathrm{s}_{1}\right)\left(\mu_{2}+\mu_{3}+\mathrm{s}_{2}\right)-\left(\mu_{1}\right.\right. \\
& \left.\left.-\mu_{2}+\mathrm{s}_{1}\right)\left(\mu_{3}-\mu_{2}+\mathrm{s}_{2}\right)\right],
\end{aligned}
$$

where $\mathrm{J}_{1}$ corresponds to the current that would be generated without GBs and lateral carrier transfer, while $\mathrm{Q}_{1}^{*}$ contains the both aforementioned influences. 
While Eqs. (A16) and (A17) can be generalized for any $\mathrm{j}$, it is not the case for Eq. (A18). Therefore, we need to apply the same procedure for $\mathrm{j}=2$ and $\mathrm{j}=3$ that leads to

$$
\begin{aligned}
\mathrm{Q}_{2}^{*}\left(\mathrm{x}_{\mathrm{ps}}, \mathrm{z}_{\mathrm{ps}}\right)= & \frac{2}{\pi} \int_{0}^{\infty}\left[\mathrm{f}_{21}(\mathrm{k}) \mathrm{e}^{\mu_{2} \mathrm{x}_{\mathrm{ps}}}\right. \\
& \left.+\mathrm{f}_{22}(\mathrm{k}) \mathrm{e}^{\mu_{2}\left(\mathrm{w}-\mathrm{x}_{\mathrm{ps}}\right)}\right] \sin \left(\mathrm{z}_{\mathrm{ps}} \mathrm{k}\right) \mathrm{dk}
\end{aligned}
$$

with

$$
\begin{aligned}
\mathrm{f}_{21}(\mathrm{k})= & \frac{\mathrm{k}}{\mu_{2}} \frac{1}{\mathrm{D}(\mathrm{k})}\left\{\mu_{3}\left(\mathrm{~s}_{2}+\mu_{3}-\mu_{2}\right)\left[\mu_{2}^{2}-\mu_{1}\left(\mathrm{~s}_{1}+\mu_{1}\right)\right]\right. \\
& \left.+\mathrm{e}^{\mathrm{w} \mu_{2}} \mu_{1}\left(\mathrm{~s}_{1}+\mu_{1}+\mu_{2}\right)\left[\mu_{3}\left(\mathrm{~s}_{2}+\mu_{3}\right)-\mu_{2}^{2}\right]\right\}
\end{aligned}
$$

and

$$
\begin{aligned}
\mathrm{f}_{22}(\mathrm{k})= & \frac{\mathrm{k}}{\mu_{2}} \frac{1}{\mathrm{D}(\mathrm{k})} \times\left\{\mu_{1}\left(\mathrm{~s}_{1}+\mu_{1}-\mu_{2}\right)\left[\mu_{2}^{2}-\mu_{3}\left(\mathrm{~s}_{2}+\mu_{3}\right)\right]\right. \\
& \left.+\mathrm{e}^{\mathrm{w} \mu_{2}} \mu_{3}\left(\mathrm{~s}_{2}+\mu_{2}+\mu_{3}\right)\left[\mu_{1}\left(\mathrm{~s}_{1}+\mu_{1}\right)-\mu_{2}^{2}\right]\right\} .
\end{aligned}
$$

The expression of $Q_{3}^{*}$ is identical to the expression of $Q_{1}^{*}$ by exchanging the indices of $\mu_{3}$ and $\mu_{1}$ as well as $\mathrm{s}_{1}$ and $\mathrm{s}_{2}$ and replacing $\mathrm{x}_{\mathrm{ps}}$ by $\left(\mathrm{w}-\mathrm{x}_{\mathrm{ps}}\right)$.

To obtain $\mathrm{h}(\mathrm{x}, \mathrm{z})$, the projection of $\mathrm{g}(\mathrm{r})$ on the $(\mathrm{x}, \mathrm{z})$ plane, we use Eq. (A5) with Eq. (5) that leads to

$$
\mathrm{h}(\mathrm{x}, \mathrm{z})=\sqrt{\pi} \sigma \mathrm{g}_{0} \exp \left(-\frac{\mathrm{x}^{2}}{\sigma^{2}}\right) \exp (-\alpha \mathrm{z}) .
$$

Making use of Eqs. (A4) and (A16) with the property of linearity of the convolution product leads to

$$
\mathrm{I}\left(\mathrm{x}_{0}\right)=\mathrm{I}_{0}\left(\mathrm{x}_{0}\right)-\sum_{\mathrm{j}=1}^{3} \mathrm{I}_{\mathrm{GB}, \mathrm{j}}\left(\mathrm{x}_{0}\right)
$$

with

$$
\mathrm{I}_{0}\left(\mathrm{x}_{0}\right)=\mathrm{q} \int_{0}^{\infty} \sum_{\mathrm{j}=1}^{3} \int_{\mathrm{x}_{\mathrm{j}-1}}^{\mathrm{x}_{\mathrm{j}}} \mathrm{J}_{\mathrm{j}}\left(\mathrm{z}_{\mathrm{ps}}\right) \times \mathrm{h}\left(\mathrm{x}_{\mathrm{ps}}-\mathrm{x}_{0}, \mathrm{z}_{\mathrm{ps}}\right) \mathrm{dx_{ \textrm {ps } }} d \mathrm{z}_{\mathrm{ps}}
$$

and

$$
\begin{aligned}
I_{G B, j}\left(x_{0}\right)= & q \int_{x_{j-1}}^{x_{j}} \int_{0}^{\infty} Q_{j}^{*}\left(x_{p s}, z_{p s}\right) \times h\left(x_{p s}\right. \\
& \left.-x_{0}, z_{p s}\right) d z_{p s} d x_{p s} .
\end{aligned}
$$

Assuming the order of integration can be permuted, the background current term $\mathrm{I}_{0}\left(\mathrm{x}_{0}\right)$ is computed relatively straightforwardly using Eqs. (A17) and (A24) in Eq. (A26), leading to

$$
\begin{aligned}
\mathrm{I}_{0}\left(\mathrm{x}_{0}\right)= & \frac{1}{2} \pi \sigma^{2} \mathrm{qg}_{0} \frac{1}{\alpha+\Lambda_{2}}\left[2 \frac{\alpha+\Lambda_{2}}{\alpha+\Lambda_{1}}-\left(\frac{\Lambda_{2}-\Lambda_{1}}{\alpha+\Lambda_{1}}\right)\right. \\
& \left.\times \operatorname{erfc}\left(-\frac{\mathrm{x}_{0}}{\sigma}\right)-\left(\frac{\Lambda_{3}-\Lambda_{2}}{\alpha+\Lambda_{3}}\right) \operatorname{erfc}\left(\frac{\mathrm{w}-\mathrm{x}_{0}}{\sigma}\right)\right] .
\end{aligned}
$$

The values obtained infinitely far from the GBs are called the plateau levels. By convention we normalize the profile to the plateau level of the left side $\left(\mathrm{I}_{01}\right)$ that is obtained by the limit of Eq. (A28) when $x_{0}$ tends to minus infinity

$$
\mathrm{I}_{01}=\pi \sigma^{2} \mathrm{qg}_{0} \frac{1}{\alpha+\Lambda_{1}} .
$$

Making use of Eqs. (A28) and (A29) and reordering leads to the normalized $\mathrm{I}_{0}$ value.

$$
\begin{aligned}
\frac{\mathrm{I}_{0}\left(\mathrm{x}_{0}\right)}{\mathrm{I}_{01}}= & \frac{1}{2}\left[2-\left(\frac{\Lambda_{2}-\Lambda_{1}}{\alpha+\Lambda_{2}}\right) \operatorname{erfc}\left(-\frac{\mathrm{x}_{0}}{\sigma}\right)-\left(\frac{\alpha+\Lambda_{1}}{\alpha+\Lambda_{2}}\right)\right. \\
& \left.\times\left(\frac{\Lambda_{3}-\Lambda_{2}}{\alpha+\Lambda_{3}}\right) \operatorname{erfc}\left(\frac{\mathrm{w}-\mathrm{x}_{0}}{\sigma}\right)\right] .
\end{aligned}
$$

Assuming the order of integration could be permutated, we compute the term $\mathrm{I}_{\mathrm{GB}, \mathrm{j}}$ from Eq. (A27) making use of Eq. (A18) and Eq. (A24) for $\mathrm{j}=1$.

$$
\begin{aligned}
\mathrm{I}_{\mathrm{GB}, 1}\left(\mathrm{x}_{0}\right)= & \mathrm{q} \sigma \mathrm{g}_{0} \frac{2}{\sqrt{\pi}} \times \int_{0}^{\infty} \mathrm{f}_{1}(\mathrm{k}) \\
& \times \int_{0}^{\infty} \mathrm{e}^{-\alpha \mathrm{z}_{\mathrm{ps}}} \sin \left(\mathrm{z}_{\mathrm{ps}} \mathrm{k}\right) \mathrm{dz}_{\mathrm{ps}} \\
& \times \int_{-\infty}^{0} \exp \left[\mu_{1} \mathrm{x}_{\mathrm{ps}}-\frac{\left(\mathrm{x}_{\mathrm{ps}}-\mathrm{x}_{0}\right)^{2}}{\sigma^{2}}\right] \mathrm{dx}_{\mathrm{ps}} \mathrm{dk} .
\end{aligned}
$$

The integration in $\mathrm{z}_{\mathrm{ps}}$ and $\mathrm{x}_{\mathrm{ps}}$ can be solved analytically resulting in, normalized by $\mathrm{I}_{01}$ [see Eq. (A29)]

$$
\begin{aligned}
\frac{\mathrm{I}_{\mathrm{GB}, 1}\left(\mathrm{x}_{0}\right)}{\mathrm{I}_{01}}= & \frac{1}{\pi}\left(\alpha+\Lambda_{1}\right) \times \int_{0}^{\infty} \mathrm{f}_{1}(\mathrm{k}) \\
& \times \frac{\mathrm{k}}{\mathrm{k}^{2}+\alpha^{2}} \times \exp \left[\left(\frac{\mu_{1} \sigma}{2}\right)^{2}+\mu_{1} \mathrm{x}_{0}\right] \\
& \times \operatorname{erfc}\left(\frac{\mu_{1} \sigma}{2}+\frac{\mathrm{x}_{0}}{\sigma}\right) \mathrm{dk} .
\end{aligned}
$$

The same procedure is used to derive the other $\mathrm{I}_{\mathrm{GB}, \mathrm{j}}$ normalized terms. The sum of all the normalized $\mathrm{I}_{\mathrm{GB}, \mathrm{j}}$ with the normalized background current [Eq. (A30)] results in the total normalized current profile expressed in Eq. (8).

\section{APPENDIX B: NUMERICAL EVALUATION OF “EXP( $\left.\mathrm{Y}^{2}\right) \operatorname{ERFC}(\mathrm{Y})$ ” TERMS}

In the case of y tending to infinity we make use of another expression for erfc(y) (Ref. 17) involving a continued fraction $[\mathrm{CF}(\mathrm{y})]$ that does not diverge 


$$
\begin{aligned}
& 2 e^{y^{2}} \int_{y}^{\infty} e^{-t^{2}} d t=\frac{1}{y+} \times \frac{1 / 2}{y+} \times \frac{1}{y+} \times \frac{3 / 2}{y+} \times \frac{2}{y+\ldots} \\
&==C F(y) \text { with } y>0 \\
& y+\frac{1}{y+\frac{1 / 2}{y+\frac{2}{y+\ldots}}}
\end{aligned}
$$

$$
\operatorname{erfc}(\mathrm{y})=\sqrt{\pi} \mathrm{e}^{-\mathrm{y}^{2}} \mathrm{CF}(\mathrm{y})
$$

Replacing this expression in "exp( $\left.\mathrm{y}^{2}\right) \operatorname{erfc}(\mathrm{y})$ " leads to Eq. (A35)

$$
\mathrm{e}^{\mathrm{y}^{2}} \operatorname{erfc}(\mathrm{y})=\sqrt{\pi} \mathrm{CF}(\mathrm{y})
$$

"exp $\left(y^{2}\right) \operatorname{erfc}(y) "$ denotes just the dependency of the exponential and erfc functions when y tends to infinity. A more general expression is, however, needed to evaluate any terms of this form thus the general case is expressed by Eq. (A36).

$$
\mathrm{e}^{\mathrm{a}} \operatorname{erfc}(\mathrm{b})=\sqrt{\pi} \mathrm{e}^{\mathrm{a}-\mathrm{b}^{2}} \mathrm{CF}(\mathrm{b}) \text { with } \mathrm{b}>0
$$

Note that if in this equation $b^{2}=a$ we find Eq. (A35). Even if Eq. (A36) works for all $b>0$, the convergence of the continued fraction is slow when $b$ is small which is the opposite case if we evaluate erfc using the Taylor series around 0 . Therefore, for practical purpose, if $|\mathrm{b}|<2$ we use the Taylor series approximation of the erfc multiplied by the exponential. For $b>2$ we use Eq. (A36) to evaluate the whole term. For $b<-2$, for speed of convergence reasons, we use the continued fraction evaluation that, however, needs to be modified to handle negative arguments. Using the property of symmetry of the erfc function of Eq. (A37) with Eq. (A36) leads to Eq. (A38).

$$
\begin{aligned}
& \operatorname{erfc}(b)=2-\operatorname{erfc}(-b) \\
& e^{a} \operatorname{erfc}(b)=2 e^{a}-\sqrt{\pi} e^{a-b^{2}} C F(-b) \quad \text { with } b<0 .
\end{aligned}
$$

The evaluation of the continued fraction $\mathrm{CF}(\mathrm{b})$ is performed using Lentz's method ${ }^{18}$ that is free of a convergence problem.

${ }^{1}$ C. H. Seager, J. Appl. Phys. 52, 3960 (1981).

${ }^{2}$ F. Meng, T. Sun, and R. Cui, Semicond. Sci. Technol. 15, 926 (2000).

${ }^{3}$ S. A. Edmiston, G. Heiser, A. B. Sproul, and M. A. Green, J. Appl. Phys. 80, 6783 (1996).

${ }^{4}$ J. Oualid, C. M. Singal, J. Dugas, J. P. Crest, and H. Amzil, J. Appl. Phys. 55, 1195 (1984).

${ }^{5}$ C. Donolato, J. Appl. Phys. 54, 1314 (1983).

${ }^{6}$ O. von Roos and K. L. Luke, J. Appl. Phys. 55, 4275 (1984).

${ }^{7}$ K. L. Luke and O. von Roos, J. Appl. Phys. 55, 2962 (1984).

${ }^{8}$ R. Corkish, T. Puzzer, and A. B. Sproul, J. Appl. Phys. 84, 5473 (1998).

${ }^{9}$ L. F. Shampine, J. Comput. Appl. Math. 211, 131 (2008).

${ }^{10}$ M. A. Green, Silicon Solar Cells: Advanced Principles \& Practice (Bridge Printery, Sydney, 1995).

${ }^{11}$ A. Mittiga and M. Capizzi, J. Appl. Phys. 62, 3443 (1987).

${ }^{12}$ G. Micard, S. Seren, and G. Hahn, Proceedings of the 23rd EU PVSEC (WIP, Munich, 2008), p. 208.

${ }^{13}$ G. Micard, S. Seren, and G. Hahn, Proceedings of the 24th EU PVSEC (WIP, Munich, 2009), p. 46.

${ }^{14}$ G. Hahn and A. Schönecker, J. Phys.: Condens. Matter 16, R1615 (2004).

${ }^{15}$ A. Mittiga, M. Capizzi, C. Coluzza, A. Frova, V. Parisi, D. Cavalcoli, L. Moro, and M. Prudenziati, J. Appl. Phys. 63, 4748 (1988).

${ }^{16}$ Wolfram Mathematica, http://www.wolfram.com/.

${ }^{17}$ M. A. Stegun and I. A. Abramowitz, Handbook of Mathematical Functions (Dover, New York, 1972).

${ }^{18}$ W. J. Lentz, Appl. Opt. 15, 668 (1976). 